\title{
Extended frequency range of transverse-electric surface plasmon polaritons in graphene
}

\author{
Zeeshan Ahmad, Egor A. Muljarov, and Sang Soon Oh $\odot^{*}$ \\ School of Physics and Astronomy, Cardiff University, Cardiff CF24 3AA, United Kingdom
}

(Received 15 June 2021; accepted 2 August 2021; published 24 August 2021)

\begin{abstract}
The dispersion relation of surface plasmon polaritons in graphene that includes optical losses is often obtained for complex wave vectors while the frequencies are assumed to be real. This approach, however, is not suitable for describing the temporal dynamics of optical excitations and the spectral properties of graphene. Here we propose an alternative approach that calculates the dispersion relation in the complex frequency and real wave vector space. This approach provides a clearer insight into the optical properties of a graphene layer and allows us to find the surface plasmon modes of a graphene sheet in the full frequency range, thus removing the earlier reported limitation $(1.667<\hbar \omega / \mu<2)$ for the transverse-electric mode. We further develop a simple analytic approximation which accurately describes the dispersion of the surface plasmon polariton modes in graphene. Using this approximation, we show that transverse-electric surface plasmon polaritons propagate along the graphene sheet without losses even at finite temperature.
\end{abstract}

DOI: 10.1103/PhysRevB.104.085426

\section{INTRODUCTION}

Surface plasmon polaritons (SPPs) are collective excitations of charge density coupled to electromagnetic waves that can travel along a conductor-dielectric interface [1]. Interestingly, even an atomically thin conducting layer, such as a graphene sheet, can support SPPs [2]. In a graphite intercalated compound that contains multiple noninteracting two-dimensional (2D) graphene layers, SPPs were modeled theoretically and observed experimentally [3]. The first discovery of graphene [4] triggered more theoretical studies on SPPs in this material [5-7]. Since the first experimental observation of SPPs in graphene [8], more efforts have been made to use the SPPs for controlling the optical properties of graphene, such as electronically tuned extraordinary transmission [9], mode confinement by gap plasmons [10], and resonant absorption by an antidot array [11].

Although most studies of SPPs have focused on transversemagnetic (TM) polarization, a single graphene layer, unlike normal-metal sheets, can also support transverse-electric (TE) SPP modes [12]. Technically, the existence of each type of SPP mode can be confirmed by solving a dispersion relation between the frequency and the wave vector following from Maxwell's equations and the conductivity model. For instance, the SPP dispersion for a dielectic/metal interface or a thin film of a Drude metal allows only TM modes which have zero magnetic field components normal to the interface and along the propagation direction. By analyzing the disper-

\section{*OhS2@cardiff.ac.uk}

Published by the American Physical Society under the terms of the Creative Commons Attribution 4.0 International license. Further distribution of this work must maintain attribution to the author(s) and the published article's title, journal citation, and DOI. sion relation for complex wave numbers and real frequencies, Mikhailov and Ziegler have shown [12] that TE SPP modes in graphene exist only in the range $1.667<\hbar \omega / \mu<2$ at zero temperature, where $\omega$ and $\mu$ are, respectively, the light frequency and the chemical potential. Here, the lower limit corresponds to the zero of the imaginary part of the optical conductivity, while the upper limit is given by the minimum of the interband transition energy at zero temperature.

Importantly, the lower limit $(\approx 1.667)$ for the normalized frequency $\hbar \omega / \mu$ of the TE SPP mode can vary because the imaginary part of the optical conductivity of graphene may change depending on temperature and gate voltage. In addition, the range for the TE SPP mode frequency $\omega$ itself can be tuned by changing the chemical potential $\mu$ which in turn may be controlled by applying gate voltage or external magnetic field [13]. In the literature, the TE SPP mode solution has been studied for a graphene layer sandwiched between two dielectric media, and it was found that the range of the TE SPP mode frequencies can be modified by changing the permittivity contrast [14]. Furthermore, the range of TE SPP modes can be reversed when the surrounding material has a negative refractive index [15]. TE SPP modes in a graphene sheet placed on top of a nonlinear material substrate were also found to be limited to a similar frequency range [16].

In this paper, we focus on the dispersion of SPP modes in a graphene layer with finite temperature and nonzero chemical potential and show that the complex-frequency analysis developed in this work removes both the upper and the lower limits for the TE SPP mode in graphene.

While it is well-known how to calculate the dispersion relations of SPPs in graphene for given optical conductivities [12], this has been done assuming that any SPP mode has a real frequency but complex wave number $q=q^{\prime}+i q^{\prime \prime}[12,16,17]$. This corresponds to a continuous-wave excitation of a SPP which has a finite propagation length within the graphene layer of the order of $1 / q^{\prime \prime}$. This picture is more suited for 
describing electromagnetic waves propagating in inhomogeneous waveguides not conserving the in-plane component of the wave number $q$. In contrast, uniform waveguides conserve $q$, which can naturally be taken real. In our approach, assuming the excitation of the system is limited in time, the temporal evolution of SPP modes is described by a complex frequency $\omega=\omega^{\prime}+i \omega^{\prime \prime}$, with typically $\omega^{\prime \prime}<0$ corresponding to a temporal decay due to radiative losses or absorption. The main advantage of this approach is that it provides direct access to the optical spectra of the system where the imaginary parts of complex frequencies of isolated modes usually correspond to the half width at half maximum of the resonance peaks. Page et al. $[18,19]$ have recently shown that the complex frequency approach can be used to describe optical gain (for $\omega^{\prime \prime}>0$ ) in a nonequilibrium inverted graphene system where TM SPP modes were considered.

The complex-frequency approach has recently become widespread and broadly used in optics, owing to the useful concept of resonant states [20,21], also known in the literature as quasinormal modes [22]. These are the eigensolutions of Maxwell's equations satisfying outgoing wave boundary conditions. They present a rigourous and powerful tool for analyzing optical spectra, such as scattering and transmission $[23,24]$, with the real part of the complex frequency of the resonant state typically corresponding to the frequency position of a spectral line and the imaginary part to the half of its linewidth. Physically, resonant states form as a result of constructive interference of multiply reflected electromagnetic waves from the boundaries or inhomogeneities within optical systems. They have been studied in the literature both in finite optical systems, such as dielectric [25-27] and plasmonic nanoparticles [28-30], and in infinitely extended systems, such as planar waveguides [31] and photonic crystals [32-34].
Although in atomically thin films, such as a single graphene layer, these states do not normally exist, the formalism of complex-frequency modes can still be very useful, as we show in this paper.

The paper is organized as follows. Section II introduces the optical conductivity of a single graphene layer. Section III A describes the secular equations determining the dispersion of SPPs in TM and TE polarizations. Sections III B and III C present the main results of the paper, including both exact numerical and approximate analytical solutions of the secular equations in the complex frequency plane, their dependence on the propagation constant, temperature, and the chemical potential, and elimination of both the lower and the upper boundaries for the TE SPP mode frequencies. The temperature dependence of the threshold frequencies is discussed in Sec. IIID. Section IV summarizes the results of the paper. Appendices A-E provide details on derivations of the optical conductivity of graphene and secular equations for the TM and TE SPP modes, and supply an additional material on our study of the TE mode near the lower threshold frequency and on the SPP dispersion at a finite damping.

\section{OPTICAL CONDUCTIVITY OF GRAPHENE}

The conductivity of graphene has an interband term in addition to the usual metallic Drude term, also referred to as intraband. It is the interband term of the conductivity which gives rise to TE SPP modes found in a graphene layer in contrast to a Drude-metal layer where such modes do not exist. Following Refs. [12,17,35], we derive in Appendices A and B the $2 \mathrm{D}$ optical conductivity of a homogeneous graphene sheet. In the long-wavelength limit, the expression for the $2 \mathrm{D}$ optical conductivity is given as a function of the light frequency $\omega$ by

$$
\sigma(\omega)=i \alpha\left\{\frac{2 \ln (2+2 \cosh \mu \beta)}{\mu \beta(\Omega+i \Gamma)}+\int_{0}^{\infty} d E[N(-E ; \mu \beta)-N(E ; \mu \beta)]\left(\frac{1}{\Omega+i \Gamma-2 E}+\frac{1}{\Omega+i \Gamma+2 E}\right)\right\},
$$

where

$$
N(E ; \xi)=\frac{1}{e^{\xi(E-1)}+1}
$$

is the Fermi-Dirac distribution, $\Omega=\hbar \omega / \mu$ is the normalized frequency, $\beta=\left(k_{B} T\right)^{-1}$ is the inverse temperature, $\alpha=$ $e^{2} /(\hbar c)$ is the fine-structure constant, and $\Gamma$ is a phenomenological damping. The first term in the curly bracket in Eq. (1) arises from intraband transitions, whereas the second one comes from interband transitions near the Dirac point in graphene dispersion. Importantly, Eq. (1) has an analytic dependence on $\Omega$ which can be continued into the complex $\Omega$-plane without changing the formula. Note that for real $\Omega$ and $\Gamma=0$, the integrand encounters a pole on the integration path. This requires that the diverging integral is split into a principal-value part and a half-pole contribution that can technically be achieved by keeping $\Gamma$ positive infinitesimal in Eq. (1). For $\operatorname{Im} \Omega \neq 0$, this is no longer needed. However, the interband term is represented by a multivalued function having a logarithmic nature. Therefore, for the analytic con- tinuation, one has to choose the right Riemann sheet which provides the proper values of the integral at real $\Omega$.

Figures 1(a) and 1(b) show the graphene conductivity as a function of normalized real frequency $\Omega=\hbar \omega / \mu$ and damping $\Gamma$ at zero temperature. Increasing temperature smoothes out the Fermi distribution, which is reflected by smearing of the steplike feature in the real part and the logarithmic divergence in the imaginary part of the interband conductivity at $\Omega=2$. The intraband Drude-like component of Eq. (1) has a zero-frequency pole which is moving away from the real axis as the damping increases. Figures 1(c) and 1(d) show cross sections of the surface plots at fixed values of $\Gamma$ and finite $(\mu \beta=10)$ and almost zero $\left(\mu \beta=10^{5}\right)$ temperatures. Due to the functional dependence of the conductivity Eq. (1) on $\Omega+i \Gamma$, Fig. 1 can also be understood as complex-frequency plots of the conductivity, treating $\Omega$ as the real and $\Gamma$ (or its portion) as the imaginary part of frequency.

In the following, we will be using, where it is convenient, the frequency $\Omega$, wave number $Q$, and temperature $(\mu \beta)^{-1}$ normalized with respect to the chemical potential $\mu$, treating the latter as a natural scaling parameter for, respectively, 

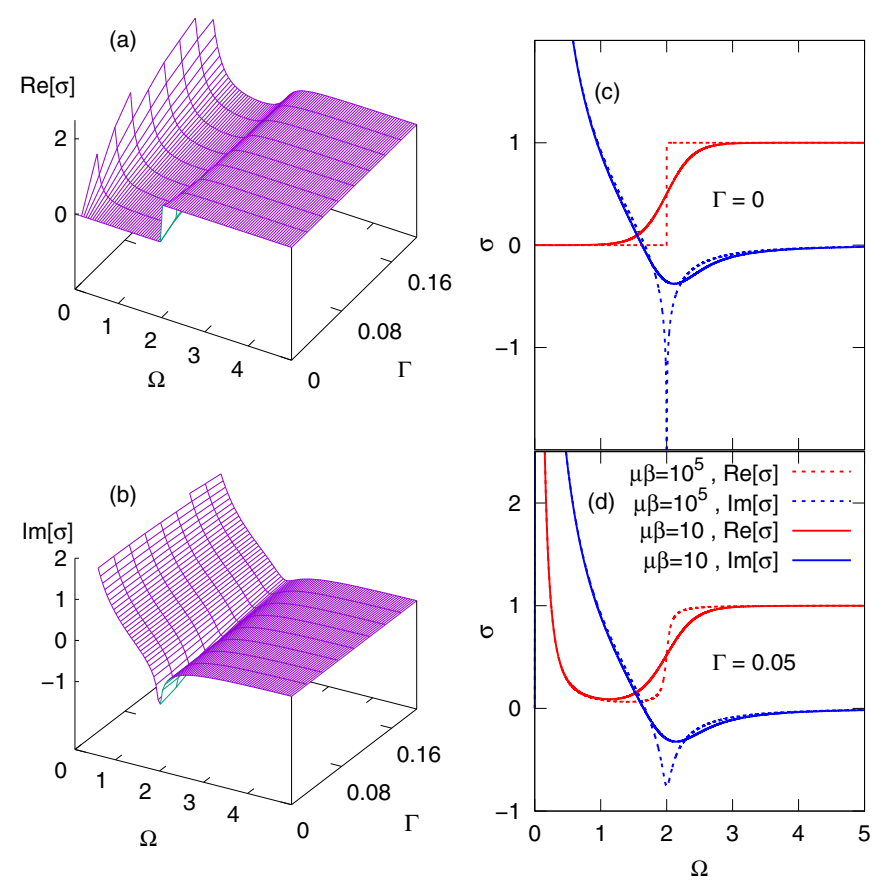

FIG. 1. Surface plots of (a) the real part $\sigma^{\prime}=\operatorname{Re} \sigma$ and (b) the imaginary part $\sigma^{\prime \prime}=\operatorname{Im} \sigma$ of the 2D optical conductivity of graphene $\sigma$ shown in units of the $\pi \alpha / 2$, where $\alpha$ is the fine-structure constant, for near zero temperature $\left(\mu \beta=10^{5}\right)$ as functions of normalized real frequency $\Omega$ and damping $\Gamma$. Dependence of $\sigma^{\prime}$ and $\sigma^{\prime \prime}$ on frequency only (c) without and (d) with damping, for $\mu \beta=10^{5}$ and $\mu \beta=10$.

the frequency $\omega$, wave number $q$, and temperature $T$. The chemical potential itself can be controlled, e.g., by charge carrier concentration of graphene [36] and is also linked to the damping $\Gamma$. In the absence of voltage or in an undoped graphene layer, the valence band is fully filled and the conduction band is empty at zero temperature, implying that $\mu=0$ and $\Gamma=0$. The concentration of free carriers-electrons in the conduction band and holes in the valence band-is zero, $n_{0}=0$. In a doped graphene layer or in the presence of voltage, the concentration of free carriers $n_{0}$ determines the chemical potential via the following equation [36,37]:

$$
n_{0}=\frac{2 \mu^{2}}{\pi(\hbar V)^{2}} \int_{0}^{\infty}[N(E ; \mu \beta)-N(E+2 ; \mu \beta)] E d E,
$$

where $V$ is the electron Fermi velocity in graphene. At zero temperature, the above integral is equal to $1 / 2$, which gives $n_{0}=\mu^{2} /\left(\pi \hbar^{2} V^{2}\right)$. At a nonzero temperature, this integral depends on $\mu$ and $T$, in accordance with Eq. (2), thus introducing a temperature-dependent correction to the above expression for $n_{0}$. The damping $\Gamma$ contributing to Eq. (1) increases with density of impurities [17].

\section{SPP MODES IN GRAPHENE}

\section{A. Secular equations for SPP modes}

In this subsection, we present the secular equations determining in each polarization the dispersion relation between the SPP mode frequency $\omega$ and the propagation wave number $q$. We briefly describe their derivation for an infinitely thin graphene sheet. To gain physical insight into SPP modes in graphene, we discuss, at the end of this subsection, a comparison between SPP modes in graphene and in a Drude metal.

For a very thin planar conducting layer, the dispersion relation of SPP modes can be obtained in two ways. One may start deriving from Maxwell's equations and boundary conditions a secular equation for the SPP modes for a finite-thickness conducting material with a bulk conductivity. This secular equation can then be simplified in the limit of an infinitesimal film thickness. Alternatively, one may obtain the secular equation and SPP dispersion relation by assuming an infinitesimal layer with a surface conductivity $\sigma(\omega)$. The two ways lead to identical results. We follow the second approach and derive in Appendix $\mathrm{C}$ the secular equations for both polarizations, assuming the conducting layer is placed at $z=0$ and is surrounded by vacuum, which is expressed by the permittivity in the entire space

$$
\varepsilon(\omega ; z)=1+\frac{2 i \sigma(\omega)}{\omega} \delta(z),
$$

where $\delta(z)$ is the Dirac delta function. Using for brevity the units in which the speed of light in vacuum $c=1$, the secular equations for SPP modes in both polarizations are given by

$$
\begin{array}{ll}
\omega+k(\omega) \sigma(\omega)=0 & (\mathrm{TM}), \\
k(\omega)+\omega \sigma(\omega)=0 & (\mathrm{TE}),
\end{array}
$$

where $k(\omega)=\sqrt{\omega^{2}-q^{2}}$ and $q$ are, respectively, the normal and in-plane components of the light wave vector in vacuum and $\omega$ is the complex light frequency. The dimensionless surface conductivity $\sigma(\omega)$ of a graphene layer to be used in the above equations is given by Eq. (1). Secular equations (5) and (6) determine the dispersion relations between the real inplane wave number $q$ and complex frequency $\omega$. Note that the same equations were used in the literature $[12,38]$ for finding SPP modes at a fixed real frequency of light, thus determining instead from Eqs. (5) and (6) complex propagation constants of SPPs.

Now, before moving on to graphene, we briefly describe the solutions to the secular equations (5) and (6) for a Drude metallic sheet. In this case, the conductivity would only consist of the first term in Eq. (1), so $\sigma(\omega) \propto i / \omega$, and Eq. (5) results in a TM mode having a square-root dispersion, $\omega \propto$ $\sqrt{q}$. We find in the next subsection a similar SPP mode for graphene in the frequency range dominated by the Drude conductivity. It can be seen that Eq. (6) for TE polarization has no solution with a nonzero real part of frequency for Drude conductivity. We found, however, a TE mode of a Drude metallic sheet which has a purely imaginary frequency: $\operatorname{Re} \omega=0$. This TE mode is also purely decaying in time: $\operatorname{Im} \omega<0$. However, with the full graphene conductivity, there is also a propagating SPP mode in TE polarization [12], with $\operatorname{Re} \omega \neq 0$, which we discuss in more depth in the rest of this section.

\section{B. SPP dispersion: Exact results and analytic approximations}

In this subsection, we present graphene SPP dispersion for both TM and TE modes which are found by solving the secular equations (5) and (6) numerically and comparing with a developed analytic approximation. We show, in particular, that the mode spectral linewidth (given by $\operatorname{Im} \omega$ ) is controlled 

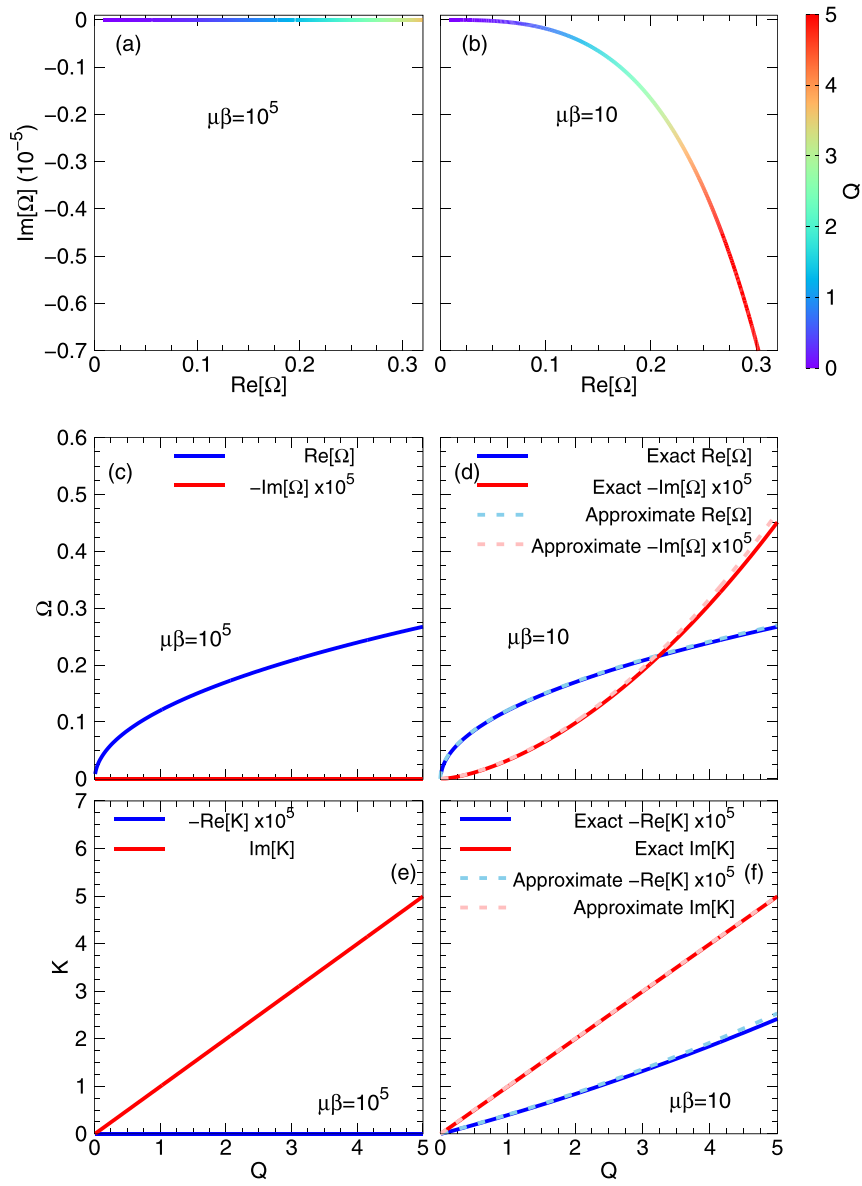

FIG. 2. (a), (b) Complex eigenfrequency $\Omega=\hbar \omega / \mu$ of TM surface plasmon mode of a graphene layer shown in the complex frequency plane as function of the real in-plane wave number of light $Q=\hbar q c / \mu$ given by the color code. Real and imaginary parts of (c), (d) the eigenfrequency $\Omega$ and (e), (f) the normal component of the light wave number $K=\hbar k c / \mu$ calculated by solving Eq. (5) exactly (solid lines) and using the approximation Eqs. (7) and (8) (dashed lines). The data is presented for (a), (c), (e) low temperature $\left(\mu \beta=10^{5}\right)$ and (b), (d), (f) high temperature $(\mu \beta=10)$, and $\Gamma=0$.

by the temperature $\mu \beta$ contributing via the Fermi function Eq. (2). We analyze the earlier reported [12] limited frequency range $1.667<\hbar \omega / \mu<2$ for the TE mode and remove both the upper and the lower boundaries for this mode.

Figure 2 shows the dispersion of the TM mode, which includes both the complex $\omega$ plots and dependencies on $q$ of the real and imaginary parts of $\omega$ and $k$, all shown at high $(\mu \beta=10)$ and low $\left(\mu \beta=10^{5}\right)$ temperatures. The TM dispersion lies well below the light line, $\omega=q$, as can be seen from Figs. 2(c) and 2(d) by comparing magnitudes of $\operatorname{Re} \omega$ and $q$. In this frequency regime, the conductivity is dominated by intraband transitions within graphene band structure, described by the Drude-like term in Eq. (1). At low temperature, both $\operatorname{Im} \omega$ and $\operatorname{Re} k$ are exponentially small, i.e., proportional to $\exp (-\mu \beta)$. Note that $\operatorname{Re} \omega$ asymptotically approaches the temperature-dependent limit $\Omega_{\mathrm{up}}^{\mathrm{TM}}$ for large $q$. The magnitude of $\operatorname{Im} \omega$ increases swiftly with $q$, with the rate determined by temperature, as more charge carrier vacancies become available below the Fermi level, compare Figs. 2(a) and 2(b). The
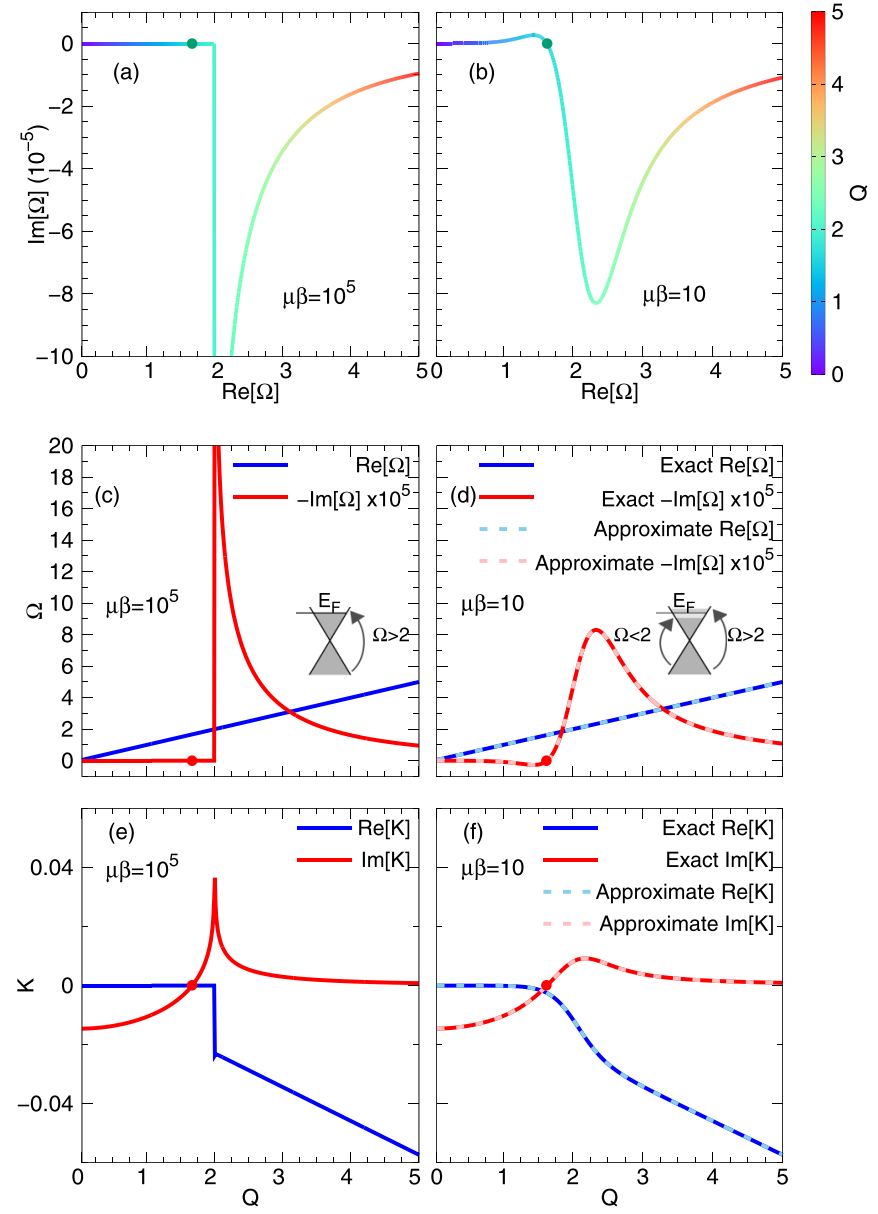

FIG. 3. As Fig. 2 but for the TE mode determined by Eq. (6) and the approximation given by Eqs. (9) and (10). Dots show the position of the lower threshold $\Omega_{\text {low }}^{\mathrm{TE}}$. Insets: Sketches of the electronic dispersion in graphene.

normal component of the wave number $k$ shown in Figs. 2(e) and 2(f) demonstrates that the SPP mode is localized in the $z$ direction, due to $\operatorname{Im} k \approx q>0$. At the same time, since $\operatorname{Re} k<0$ there is a propagation of light toward the conducting sheet, although the propagation constant $|\operatorname{Re} k|$ is much smaller than $q$.

We also show in Figs. 2(d) and 2(f) by dashed lines the following approximate relations derived in Appendix $\mathrm{C} 1$ :

$$
\begin{gathered}
\omega(q) \approx \sqrt{\omega_{0} q}-i q \sigma^{\prime} / 2, \\
k(q) \approx-\sqrt{\omega_{0} q} \sigma^{\prime} / 2+i q,
\end{gathered}
$$

where $\sigma^{\prime}$ is the real part of the conductivity (taken at the mode frequency, $\left.\omega \approx \sqrt{\omega_{0} q}\right)$ and $\omega_{0}=2 \alpha \ln (2+2 \cosh \mu \beta) /(\hbar \beta)$. The approximation is based on the fact that in this frequency range, the conductivity is dominated by the Drude term, so its imaginary part is given by $\sigma^{\prime \prime} \approx \omega_{0} / \omega$ whereas the real part can be treated as a small correction, i.e., $\left|\sigma^{\prime}\right| \ll\left|\sigma^{\prime \prime}\right|$.

Figure 3 presents a dispersion of the TE mode in graphene, again showing both the complex $\omega$ plots and dependencies $\omega(q)$ and $k(q)$. The SPP mode in this polarization of light is unique to the graphene conductivity [12] due to the interband part of Eq. (1) and does not exist in a normal (e.g., Drude) 
metallic sheet. The real part of the dispersion curve, $\operatorname{Re} \omega$, lies close to the light line $\omega=q$, while the imaginary part, $\operatorname{Im} \omega$, is a few orders of magnitude smaller than the real part, as is clear from Figs. 3(c) and 3(d). Interestingly, both the real and imaginary parts of $k$, albeit being small compared to $q$, are now comparable to each other, in contrast to the TM SPP mode [compare Figs. 2(f) and 3(f)].

An approximate analytic solution was also obtained for the TE mode by using the fact that away from the $\omega=0$ pole, the graphene conductivity is small, $|\sigma(\omega)| \ll 1$, as it is proportional to the fine-structure constant which is a small number, $\alpha \ll 1$. In this limit, the solution to Eq. (6) takes the form

$$
\begin{gathered}
\omega(q) \approx q+i q \sigma^{\prime} \sigma^{\prime \prime}, \\
k(q) \approx-q \sigma^{\prime}-i q \sigma^{\prime \prime},
\end{gathered}
$$

where $\sigma^{\prime}$ and $\sigma^{\prime \prime}$ are both taken at $\omega=q$, see Appendix C 2 for derivation. The approximate complex frequency $\omega(q)$ and wave number $k(q)$ are plotted as dashed curves in Figs. 3(d) and 3(f), showing excellent agreement with the exact solution.

For completeness, we also show in Appendix E the SPP mode dispersion both in TM and TE polarizations for the graphene conductivity at a nonzero damping of $\Gamma=0.05$.

\section{Removing the boundaries for the TE mode}

The TE mode dispersion in graphene has been studied in Ref. [12] at zero temperature, with the mode frequency reaching but never exceeding the upper boundary at $\Omega_{\mathrm{up}}^{\mathrm{TE}}=2$. Our complex-frequency analysis allows us to eliminate this boundary, even for low temperatures. In fact, we see from Fig. 3 that the TE mode exists both below $\left(\operatorname{Re} \Omega<\Omega_{\text {up }}^{\mathrm{TE}}\right.$ ) and above $\left(\operatorname{Re} \Omega>\Omega_{\mathrm{up}}^{\mathrm{TE}}\right)$ the threshold. Furthermore, we observe, by comparing Figs. 3(c) and 3(d), that the temporal loss, which is given by $-\operatorname{Im} \Omega$, increases (decreases) with temperature below (above) the threshold. This can be understood simply as a smearing effect of the electronic distribution over the graphene band structure as temperature rises. Note that the threshold frequency is exactly twice the Fermi level $E_{F}$ of graphene, see the insets in Figs. 3(c) and 3(d) which provide sketches of the electronic dispersion. At zero temperature, interband absorption only takes place above $\Omega_{\mathrm{up}}^{\mathrm{TE}}$ since no charge carriers occupy the electronic bands above the Fermi level, and so the losses are high for $\operatorname{Re} \Omega>\Omega_{\mathrm{up}}^{\mathrm{TE}}$ and zero for $\operatorname{Re} \Omega<\Omega_{\text {up }}^{\mathrm{TE}}$. When temperature is finite, some charge carriers occupy energy states above the Fermi level, thus the interband absorption decreases for $\operatorname{Re} \Omega>\Omega_{\text {up }}^{\mathrm{TE}}$, in this way reducing the losses. At the same time, vacancies of charge carriers are formed below the Fermi level at finite temperature and interband absorption can take place also for $\operatorname{Re} \Omega<\Omega_{\mathrm{up}}^{\mathrm{TE}}$, so for this region the losses increase with temperature. This increase of losses is also reflected by the real part of the conductivity. In fact, it is clear from Fig. 1(c) that the real part is smeared around $\operatorname{Re} \Omega=\Omega_{\mathrm{up}}^{\mathrm{TE}}$ as the temperature increases. No similar effects are observed for the TM mode.

Figure 3 also demonstrates the earlier reported in the literature [12] lower threshold $\Omega_{\text {low }}^{\mathrm{TE}}$ for the TE mode, which was observed at $\Omega_{\text {low }}^{\mathrm{TE}} \approx 1.667$ at zero $T$. It is shown in Fig. 3 by the dots in the complex frequency plane, on the imaginary part of the mode frequency $\omega$ and the normal component of the wave number $k$. Both imaginary parts change their sign at this threshold due to the change of sign of $\sigma^{\prime \prime}$ [see Eqs. (9) and (10) and also Fig. 1(c)]. Physically, this threshold frequency corresponds to a condition that the intraband and interband electronic transitions are in balance. From a technical viewpoint, however, a positive imaginary part of the frequency, $\operatorname{Im} \omega>0$, observed below the threshold, implies an exponential growth in time of the electric and magnetic fields (at any given point in space), whereas a negative imaginary part of the wave number, $\operatorname{Re} k<0$, also observed below the threshold, means an exponential growth of the field in space away from the graphene layer. While the latter is typical for radiative modes [21] and thus seems acceptable, the former usually corresponds to a gain [30], which is obviously not present in this system. One could therefore conclude that the TE mode does not exist below the threshold. However, the complex-frequency analysis allows us again to understand the properties of the SPP mode near the threshold and to eliminate this lower boundary.

To see that the TE mode exists both above and below the lower threshold at $\Omega_{\text {low }}^{\mathrm{TE}}$, we consider the spatial and temporal behavior of the electric field together, having the following explicit form $E(x, z ; t)=E_{0} e^{i(q x+k z-\omega t)}$, where $E_{0}$ is a constant, and we have taken $z>0$ for definiteness (see Appendix C for the analytic form of the fields). Now, separating the real and imaginary parts of the frequency, $\omega=\omega^{\prime}+i \omega^{\prime \prime}$, and of the normal component of the wave number, $k=k^{\prime}+i k^{\prime \prime}$, and using the fact that the in-plane component of the wave number $q$ is real, we can separate the oscillating part of the field, $E_{0} e^{i\left(q x+k^{\prime} z-\omega^{\prime} t\right)}$, from its amplitude,

$$
|E(x, z ; t)|=\left|E_{0}\right| e^{-\left(k^{\prime \prime} z-\omega^{\prime \prime} t\right)},
$$

which is either exponentially decaying or exponentially growing in time and space. Using the fact that $\sigma^{\prime}>0$, which is equivalent to the positive imaginary part of the permittivity, we find from Eq. (10) that $k^{\prime}<0$, which physically corresponds to a plane wave propagating in vacuum toward the graphene sheet. The in-plane wave number $q$ is, however, much larger, $q \gg\left|k^{\prime}\right|$, so the direction of the electromagnetic wave is almost parallel to the sheet. At the same time, the $z$ coordinate of any point sitting on the wavefront and moving together with it can be approximately described as a function of time by $z(t)=z(0)+k^{\prime} t / q$. Substituting this into Eq. (11), we find the amplitude of the field at the selected point on the wavefront to be

$$
A(t)=A(0) e^{-k^{\prime \prime} z(t)+\omega^{\prime \prime} t}=\tilde{A}(0) e^{-\gamma t},
$$

where we have introduced a temporal decay rate:

$$
\gamma=\frac{k^{\prime \prime} k^{\prime}}{q}-\omega^{\prime \prime}
$$

Now, substituting here the dispersion for the TE mode given by Eqs. (9) and (10), we find

$$
\gamma_{\mathrm{TE}}=0 \text {, }
$$

which implies that the TE mode has no losses in reality. In contrast, for the Drude-like TM mode, the decay rate found from Eq. (13) and the approximation Eqs. (7) and (8) is given by

$$
\gamma_{\mathrm{TM}}=\sigma^{\prime}\left(q-\sqrt{\omega_{0} q}\right) / 2>0
$$




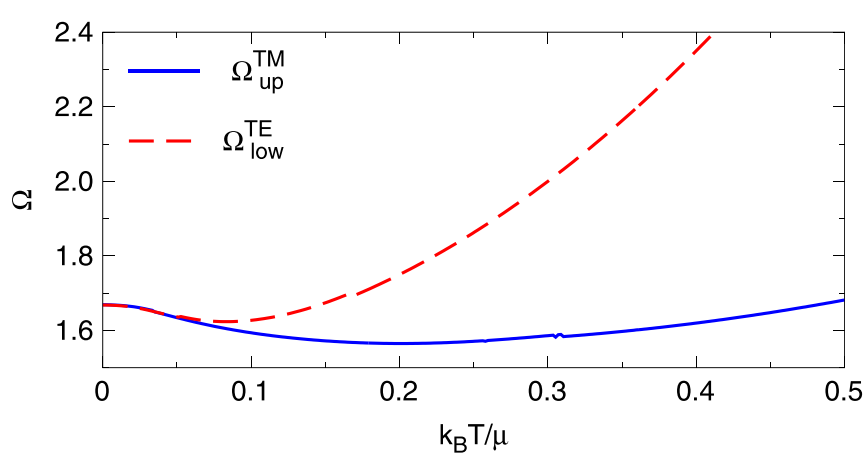

FIG. 4. Lower threshold frequency $\Omega_{\mathrm{low}}^{\mathrm{TE}}$ for the TE mode (red dashed line) and upper real frequency limit $\Omega_{\mathrm{up}}^{\mathrm{TM}}$ for the TM mode (blue solid line) as functions of the normalized temperature $(\mu \beta)^{-1}=k_{B} T / \mu$.

which implies an absorption, since the amplitude $A(t)$ of the wave front decays with time in this case. However, for a Drude conductivity without damping, we obtain $\gamma_{\mathrm{TM}}=0$, since the real part of the conductivity $\sigma^{\prime}$ vanishes, again implying that there are no losses in the system. The same is true for the graphene conductivity at zero temperature, since in this case $\sigma^{\prime}=0$ for $\Omega<2$. We would like to emphasize, however, that Eq. (14) is obtained for the TE mode at a nonzero temperature when losses are present in the conductivity, since $\sigma^{\prime}>0$ (equivalent to $\operatorname{Im} \varepsilon>0$ ).

We see that the result for the TE mode, Eq. (14), is the same below and above the lower threshold at $\Omega_{\text {low }}^{\mathrm{TE}}$, and the TE mode demonstrates a fully physical behavior from the energy conservation viewpoint on both sides of the threshold, as discussed above. Note, however, that below the threshold, the TE mode demonstrates a unique behavior, not usually observed in open systems: With no gain in the system, the electromagnetic field exponentially grows with time, albeit at a very small rate compared to the mode frequency.

\section{Temperature dependence of the threshold frequencies}

Finally, we study the temperature dependence of the TE threshold frequency, $\Omega_{\mathrm{low}}^{\mathrm{TE}}$. As discussed earlier in relation to Eqs. (9) and (10), this frequency threshold corresponds to a simultaneously change of sign of the imaginary part of the conductivity and the mode frequency. The red dashed line in Fig. 4 showing the threshold frequency $\Omega_{\text {low }}^{\mathrm{TE}}$ as a function of temperate is thus a solution of the equation $\operatorname{Im} \sigma(\omega)=0$. Figure 4 clearly demonstrates that the threshold frequency has a minimum, taking the value of $\Omega_{\text {low }}^{\mathrm{TE}}=1.6225$ at $1 / \mu \beta=$ 0.0824 , which is the result of a trade-off of the two terms in the graphene conductivity, corresponding to the intraband and interband transitions. More details on the properties of the TE mode near the lower threshold are provided in Appendix $\mathrm{D}$, where an analytic equation determining its value at zero temperature $\left(\Omega_{\text {low }}^{\mathrm{TE}}=1.667\right)$ is derived.

Interestingly, the TM modes has an upper threshold $\Omega_{\mathrm{up}}^{\mathrm{TM}}$ which coincides with $\Omega_{\text {low }}^{\mathrm{TE}}$ at zero temperature but deviates for nonzero temperatures. It is also shown in Fig. 4 by a blue solid curve. This threshold, however, has a different physical meaning as it plays the role of an asymptote for the TM mode frequency in the limit of $q \rightarrow \infty$. It can be seen that in this limit,
Eq. (5) simplifies to $\sigma(\omega)=0$. Clearly, at zero temperature and frequencies below $\Omega_{\mathrm{up}}^{\mathrm{TE}}=2$, this coincides with the equation $\operatorname{Im} \sigma(\omega)=0$ for $\Omega_{\text {low }}^{\mathrm{TE}}$, as can be seen also from Fig. 1(a), demonstrating that $\operatorname{Re} \sigma(\omega)=0$ in this frequency range.

\section{CONCLUSIONS}

In summary, we have numerically calculated the complexfrequency dispersion of the SPP modes in a homogeneous graphene layer both in TM and TE polarizations of light. We have further developed a simple analytic approximation which agrees well with the numerically exact solution of the secular equations for the modes in both polarizations.

We have shown that the TM SPP mode is determined by the Drude-like intraband part of the optical conductivity, demonstrating a square-root dispersion of the mode frequency with respect to the propagation wave number. In this polarization, the temporal decay of the electromagnetic field, which is given by the imaginary part of the mode frequency, monotonously increases with temperature. The TE SPP mode is, in turn, determined by both the intraband and the interband part, the latter being crucial for its existence. Unlike the TM mode, its dispersion is close to the light line, and the temporal decay demonstrates a nontrivial dependence on temperature and the propagation wave number. We have observed, in particular, that at finite temperature and chemical potential the TE mode exists above the upper threshold for the normalized frequency at $\Omega_{\mathrm{up}}^{\mathrm{TE}}=2$, posed by an asymptotic behavior of the dispersion at zero temperature. The temporal decay rate of the TE mode increases below the threshold and decreases otherwise as the temperature increases. This is explained by considering occupation of electronic energy bands in graphene near the K-point at different temperatures.

We have also proven that the TE mode exists both above and below the lower threshold $\Omega=\Omega_{\text {low }}^{\mathrm{TE}}$ (taking the value of $\Omega_{\text {low }}^{\mathrm{TE}}=1.667$ at zero temperature) and have studied its behavior near the threshold. This threshold is caused by a change of the sign of the imaginary part of the graphene conductivity which, in turn, causes a simultaneous change of the sign of the imaginary part of the SPP complex eigenfrequency and the normal component of the wave number. By investigating its spatial and temporal evolution, we have shown that the TE SPP in graphene presents a unique optical mode, as it can have below the threshold a positive imaginary part of the eigenfrequency without introducing gain into the material. Furthermore, we have demonstrated that in spite of the positive real part of the conductivity implying the positive imaginary part of the permittivity and hence an absorption, the TE SPP mode propagates along the graphene sheet without losses even at nonzero temperatures. This is correct at least up to second order in the conductivity.

\section{ACKNOWLEDGMENTS}

Z.A. acknowledges financial support of EPSRC under the DTP scheme. S.S.O. acknowledges the support of Sêr Cymru II Rising Star Fellowship [80762-CU145 (East)], which is partly funded by the European Regional Development Fund (ERDF) via the Welsh Government. 


\section{APPENDIX A: INTRABAND CONDUCTIVITY OF GRAPHENE}

Following Refs. [12,17,35], one can derive the 2D optical conductivity of a homogeneous graphene sheet by using the Kubo formula [6,39-41], namely, by expanding the thermodynamic average of the current to first order in the amplitude of an external electric field. In the long wavelength limit, i.e., for a small light wave number compared to that of the electron, one may neglect effects of the spatial dispersion. The intraband conductivity is then given by [12]:

$$
\sigma_{\alpha \beta}^{\text {intra }}(\omega)=-\frac{i e^{2} g_{s} g_{v}}{\hbar^{2} \omega S} \sum_{\mathbf{k} l} \frac{\partial E_{\mathbf{k} l}}{\partial k_{\alpha}} \frac{\partial f\left(E_{\mathbf{k} l}\right)}{\partial E_{\mathbf{k} l}} \frac{\partial E_{\mathbf{k} l}}{\partial k_{\beta}},
$$

where $g_{s}=2$ and $g_{v}=2$ are, respectively, the spin and valley degeneracies, $S$ is the sample area,

$$
E_{\mathbf{k} l}=(-1)^{l} \hbar V k=(-1)^{l} \hbar V \sqrt{k_{x}^{2}+k_{y}^{2}}
$$

is the electron dispersion in graphene, with $l=1$ and 2,

$$
f(E)=\frac{1}{1+e^{\beta(E-\mu)}}
$$

is the Fermi-Dirac distribution function, and $V$ is the electron Fermi velocity. Since we neglect the spatial dispersion, the conductivity tensor has a diagonal symmetric form, $\sigma_{x x}=$ $\sigma_{y y}=\sigma$ with $\sigma_{x y}=\sigma_{y x}=0$. The expressions for the partial derivatives are given by

$$
\frac{\partial E_{\mathbf{k} l}}{\partial k_{\alpha}}=(-1)^{l} \hbar V \frac{k_{\alpha}}{k},
$$

which we substitute into Eq. (A1) to obtain

$$
\begin{aligned}
\sigma^{\text {intra }}(\omega) & =\frac{-4 i e^{2}}{\hbar^{2} \omega(2 \pi)^{2}} \iint d k_{x} d k_{y} \hbar^{2} V^{2} \frac{k_{x}^{2}}{k^{2}} \sum_{l=1}^{2} \frac{\partial f\left(E_{\mathbf{k} l}\right)}{\partial E_{\mathbf{k} l}} \\
& =-\frac{i e^{2}}{\pi \hbar^{2} \omega} \int_{0}^{\infty} \frac{\partial g(E)}{\partial E} E d E,
\end{aligned}
$$

after introducing $g(E)=f(E)-f(-E)$ and performing integration over the angle in polar coordinates. The last integral can be evaluated analytically using integration by parts,

$$
\int_{0}^{\infty} \frac{\partial g(E)}{\partial E} E d E=\left.E g(E)\right|_{0} ^{\infty}-\int_{0}^{\infty} g(E) d E=\left.G(E)\right|_{0} ^{\infty},
$$

where

$$
G(E)=E[f(E)-f(-E)]-\frac{1}{\beta} \ln [f(E) f(-E)],
$$

which follows from the fact that for the Fermi function,

$$
\frac{\partial f(E)}{\partial E}=\beta f(E)[f(E)-1] .
$$

Applying the limits of integration, we obtain

$$
\begin{gathered}
G(0)=\frac{1}{\beta} \ln \left(1+e^{-\beta \mu}\right)^{2}, \\
G(\infty)=\lim _{E \rightarrow \infty}\left[-E+\frac{1}{\beta} \ln e^{\beta(E-\mu)}\right]=-\mu,
\end{gathered}
$$

So

$$
G(\infty)-G(0)=-\frac{1}{\beta} \ln [2+2 \cosh (\beta \mu)]
$$

and, finally,

$$
\sigma^{\text {intra }}(\omega)=\frac{i e^{2} \ln [2+2 \cosh (\beta \mu)]}{\pi \hbar^{2} \beta \omega} .
$$

At zero temperature, $\mu \beta \rightarrow \infty$, the intraband conductivity simplifies to

$$
\sigma^{\text {intra }}=\frac{i e^{2} \mu}{\pi \hbar^{2} \omega} .
$$

\section{APPENDIX B: INTERBAND CONDUCTIVITY OF GRAPHENE}

The interband conductivity is derived in a similar way, i.e., again using the Kubo formula, which leads in the long wavelength limit to the following expression [12]:

$$
\begin{aligned}
\sigma_{\alpha \beta}^{\text {intra }}(\omega)= & \frac{i e^{2} \hbar g_{s} g_{v}}{S} \sum_{\mathbf{k}, l \neq l^{\prime}} \frac{f\left(E_{\mathbf{k} l^{\prime}}\right)-f\left(E_{\mathbf{k} l}\right)}{E_{\mathbf{k} l^{\prime}}-E_{\mathbf{k} l}-\hbar\left(\omega+i 0_{+}\right)} \\
& \times \frac{1}{E_{\mathbf{k} l^{\prime}}-E_{\mathbf{k} l}}\left\langle\mathbf{k} l\left|\hat{v}_{\alpha}\right| \mathbf{k} l^{\prime}\right\rangle\left\langle\mathbf{k} l^{\prime}\left|\hat{v}_{\beta}\right| \mathbf{k} l\right\rangle,
\end{aligned}
$$

where $0_{+}$is a positive infinitesimal, and

$$
\hat{v}_{\alpha}=V \hat{\sigma}_{\alpha},
$$

with $\hat{\sigma}_{\alpha}$ being the Pauli matrix. $|\mathbf{k} l\rangle$ are the eigenstates of the electronic Hamiltonian near the $K$-point in the Brillouin zone,

$$
\hat{H}=V \hat{\boldsymbol{\sigma}} \cdot \hat{\mathbf{p}},
$$

corresponding to its eigenvalues Eq. (A2) and having the following explicit form:

$$
|\mathbf{k} l\rangle=\frac{1}{\sqrt{2} k}\left(\begin{array}{c}
k_{x}-i k_{y} \\
(-1)^{l} k
\end{array}\right) .
$$

The matrix elements in Eq. (B1) then take the form

$$
\left\langle\mathbf{k} 1\left|\hat{v}_{x}\right| \mathbf{k} 2\right\rangle=\frac{i k_{y}}{k} V, \quad\left\langle\mathbf{k} 1\left|\hat{v}_{y}\right| \mathbf{k} 2\right\rangle=-\frac{i k_{x}}{k} V .
$$

Again using the symmetry of the conductivity tensor in the absence of the spatial dispersion, we obtain with the help of Eq. (A2) and after integration over the angle in polar coordinates:

$$
\begin{aligned}
\sigma^{\text {inter }}(\omega)= & \frac{4 i e^{2} \hbar}{(2 \pi)^{2}} \iint d k_{x} d k_{y} V^{2} \frac{k_{y}^{2}}{k^{2}} \sum_{l=1}^{2} \frac{1}{E_{\mathbf{k} l^{\prime}}-E_{\mathbf{k} l}} \\
& \times \frac{f\left(E_{\mathbf{k} l^{\prime}}\right)-f\left(E_{\mathbf{k} l}\right)}{E_{\mathbf{k} l^{\prime}}-E_{\mathbf{k} l}-\hbar\left(\omega+i 0_{+}\right)} \\
= & \frac{i e^{2}}{2 \pi \hbar} \int_{0}^{\infty} d E[f(-E)-f(E)] \\
& \times\left[\frac{1}{\hbar\left(\omega+i 0_{+}\right)-2 E}+\frac{1}{\hbar\left(\omega+i 0_{+}\right)+2 E}\right] .
\end{aligned}
$$




\section{APPENDIX C: SPP MODES OF A THIN CONDUCTING SHEET IN VACUUM}

To derive the secular equations (5) and (6) for the SPP modes in a graphene layer, let us consider a model of an infinitely thin sheet with 2D optical conductivity $\sigma(\omega)$ placed at $z=0$. Choosing $x$ as the propagation direction of light, so the parallel components of the wave number are $k_{x}=q$ and $k_{y}=0$, Maxwell's equations are split into two blocks of first-order partial differential equations, separating TM and TE polarizations [34],

$$
\begin{array}{r}
\text { TM: }\left(\begin{array}{ccc}
\omega \mu(\omega ; z) & -\partial_{z} & i q \\
\partial_{z} & \omega \varepsilon(\omega ; z) & 0 \\
-i q & 0 & \omega \varepsilon(\omega ; z)
\end{array}\right)\left(\begin{array}{c}
i H_{y} \\
E_{x} \\
E_{z}
\end{array}\right)=0, \\
\text { TE: }\left(\begin{array}{ccc}
\omega \varepsilon(\omega ; z) & -\partial_{z} & i q \\
\partial_{z} & \omega \mu(\omega ; z) & 0 \\
-i q & 0 & \omega \mu(\omega ; z)
\end{array}\right)\left(\begin{array}{c}
E_{y} \\
i H_{x} \\
i H_{z}
\end{array}\right)=0,
\end{array}
$$

where the speed of light in vacuum is taken $c=1$ for brevity, $\partial_{z} \equiv \partial / \partial z, \varepsilon(\omega ; z)$ and $\mu(\omega ; z)$ are, respectively, the frequency and spatially dependent permittivity and permeability, $\boldsymbol{E}=$ $A_{0}\left(E_{x}, E_{y}, E_{z}\right)$ and $\boldsymbol{H}=A_{0}\left(H_{x}, H_{y}, H_{z}\right)$ are, respectively, the electric and magnetic fields, $\omega$ is the light frequency, and $A_{0}(x, t)=e^{i(q x-\omega t)}$ is a common factor representing the temporal behavior and the spatial dependence of the fields in the propagation direction. Clearly, Eqs. (C1) and (C2) can be obtained from each other by simultaneous swapping $\varepsilon \leftrightarrow \mu$ and $\boldsymbol{E} \leftrightarrow i \boldsymbol{H}$ [34].

Let us further assume that

$$
\begin{gathered}
\mu(\omega ; z)=1, \\
\varepsilon(\omega ; z)=1+\chi(\omega) \delta(z),
\end{gathered}
$$

where

$$
\chi(\omega)=\frac{2 i \sigma(\omega)}{\omega}
$$

is a $2 \mathrm{D}$ susceptibility and $\sigma(\omega)$ is the $2 \mathrm{D}$ electrical conductivity of the graphene sheet,

$$
\sigma(\omega)=2 \pi\left[\sigma^{\text {intra }}(\omega)+\sigma^{\text {inter }}(\omega)\right],
$$

consisting of the intraband and interband components calculated in Appendices A and B. Note that the factor of 2 affecting the definition of $\sigma$ is introduced in Eq. (C5) for convenience.

\section{TM polarization} fies to

The set of equations (C1) for TM polarization then simpli-

$$
\begin{gathered}
i \omega H_{y}-\partial_{z} E_{x}+i q E_{z}=0, \\
i \partial_{z} H_{y}+\omega \varepsilon E_{x}=0, \\
q H_{y}+\omega \varepsilon E_{z}=0 .
\end{gathered}
$$

For $z \neq 0$, we find the wave equation for $H_{y}$ and relations between the field components:

$$
\partial_{z}^{2} H_{y}+\left(\omega^{2}-q^{2}\right) H_{y}=0,
$$

$$
\begin{gathered}
E_{x}=-\frac{i}{\omega} \partial_{z} H_{y}, \\
E_{z}=-\frac{q}{\omega} H_{y} .
\end{gathered}
$$

To include the $z=0$ point, we use Eq. (C4) and integrate Eqs. (C7), (C8), and (C9) over an infinitesimal interval including $z=0$. Then we obtain

$$
\begin{gathered}
E_{x}\left(0_{+}\right)-E_{x}\left(0_{-}\right)=0, \\
-H_{y}\left(0_{+}\right)+H_{y}\left(0_{-}\right)+i \omega \chi E_{x}(0)=0, \\
\omega \chi E_{z}(0)=0
\end{gathered}
$$

where $0_{+}\left(0_{-}\right)$is a positive (negative) infinitesimal. From the above Eqs. (C10) - (C15) we find

$$
\begin{gathered}
H_{y}=A \operatorname{sgn}(z) e^{i k|z|}, \\
E_{x}=A \frac{k}{\omega} e^{i k|z|}, \\
E_{z}=-A \frac{q}{\omega} \operatorname{sgn}(z) e^{i k|z|},
\end{gathered}
$$

after applying outgoing wave boundary conditions to the electromagnetic field. Here $A$ is a normalization constant,

$$
\operatorname{sgn}(z)= \begin{cases}1, & z>0 \\ 0, & z=0 \\ -1, & z<0\end{cases}
$$

and $k$ is the normal component of the wave number in vacuum satisfying the light dispersion:

$$
\omega^{2}=k^{2}+q^{2} .
$$

Finally, using Eq. (C14), we obtain a secular equation for the SPP mode,

$$
i k \chi=2 \text {, }
$$

which can be written more explicitly, using Eq. (C5), as

$$
k \sigma(\omega)+\omega=0,
$$

identical to Eq. (5).

To obtain an approximate analytic solution to Eq. (C22), let us first consider the limiting case of very small frequencies when the conductivity is dominated by intraband transitions. This results in the standard SPP mode of an undamped Drude metal sheet. In fact, in this case,

$$
\sigma(\omega) \approx 2 \pi \sigma^{\text {intra }}(\omega)=\frac{i \omega_{0}}{\omega}
$$

where

$$
\omega_{0}=2 \alpha \frac{\ln (2+2 \cosh \mu \beta)}{\hbar \beta},
$$

see Eq. (1) (at zero temperature $\omega_{0}$ simplifies to just $\omega_{0}=$ $2 \alpha \mu / \hbar)$. Then the solution of Eq. (C22) takes the form

$$
\bar{k}=i \varkappa, \quad \bar{\omega}=\sqrt{\varkappa \omega_{0}},
$$

where

$$
\varkappa=\sqrt{q^{2}+\omega_{0}^{2} / 4}-\omega_{0} / 2 .
$$


Now, taking the full 2D conductivity of graphene, Eq. (C6), and treating $\Delta \sigma=2 \pi \sigma^{\text {inter }}$ as a correction to the Drude conductivity, Eq. (C23), results in a refinement of the SPP mode wave number and frequency, $k=\bar{k}+\Delta k$ and $\omega=\bar{\omega}+\Delta \omega$. We find in particular from Eqs. (C20) and (C22):

$$
-(i \varkappa+\Delta k)\left(i \omega_{0}+\omega \Delta \sigma\right)=\omega^{2}=(i \varkappa+\Delta k)^{2}+q^{2},
$$

and keeping in the above equation only terms linear in $\Delta k$ and $\Delta \sigma$, obtain

$$
\Delta k \approx-\frac{\varkappa \bar{\omega}}{2 \varkappa+\omega_{0}} \Delta \sigma
$$

Then, from Eq. (C20) we find

$$
\bar{\omega} \Delta \omega \approx \bar{k} \Delta k,
$$

which results in

$$
\Delta \omega \approx-i \frac{\varkappa^{2}}{2 \varkappa+\omega_{0}} \Delta \sigma .
$$

Finally, in the limit $q \gg \omega_{0}$, we obtain

$$
\begin{gathered}
\varkappa \approx q, \quad \bar{\omega} \approx \sqrt{q \omega_{0}}, \\
\omega^{\prime} \approx \bar{\omega}, \quad \omega^{\prime \prime} \approx-\frac{q}{2} \sigma^{\prime}(\bar{\omega}), \\
k^{\prime} \approx-\frac{\bar{\omega}}{2} \sigma^{\prime}(\bar{\omega}), \quad k^{\prime \prime} \approx q,
\end{gathered}
$$

separating the real and imaginary parts of $k=k^{\prime}+i k^{\prime \prime}, \omega=$ $\omega^{\prime}+i \omega^{\prime \prime}$, and $\sigma=\sigma^{\prime}+i \sigma^{\prime \prime}$ and neglecting $\operatorname{Im} \sigma^{\text {inter }}$ which is small compared to the Drude term.

\section{TE polarization}

The secular equation for TE polarization is obtained in a similar way. Using Eqs. (C3) and (C4), the TE block given by Eq. (C2) can be written as

$$
\begin{gathered}
\omega \varepsilon E_{y}-i \partial_{z} H_{x}-q H_{z}=0, \\
\partial_{z} E_{y}+i \omega H_{x}=0, \\
-q E_{y}+\omega H_{z}=0 .
\end{gathered}
$$

For $z \neq 0$, we obtain

$$
\begin{gathered}
\partial_{z}^{2} E_{y}+\left(\omega^{2}-q^{2}\right) E_{y}=0, \\
H_{x}=\frac{i}{\omega} \partial_{z} E_{y}, \\
H_{z}=\frac{q}{\omega} E_{y},
\end{gathered}
$$

and for $z=0$, we integrate Eq. (C34) around this point, obtaining

$$
H_{x}\left(0_{+}\right)-H_{x}\left(0_{-}\right)=-i \omega \chi E_{y}(0),
$$

at the same time having both $E_{y}$ and $H_{z}$ continuous across $z=$ 0 . A solution satisfying outgoing or incoming wave boundary conditions then takes the form

$$
E_{y}(z)=A e^{i k|z|},
$$

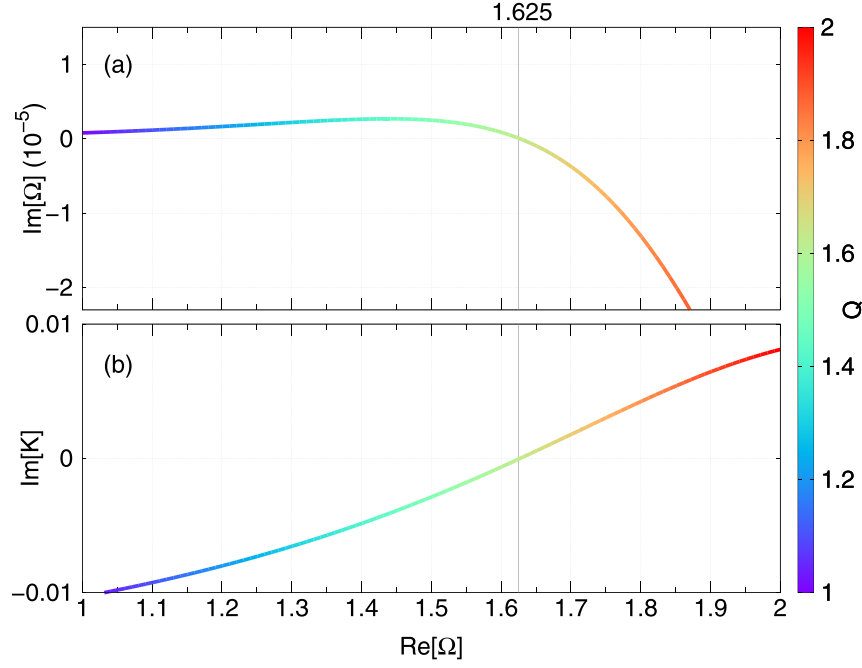

FIG. 5. Imaginary part of (a) the TE mode frequency $\Omega$ and (b) the normal component of the light wave number $K$, as functions of the real part of $\Omega$, calculated for $\mu \beta=10$ for changing in-plane light wave number $Q$ given by the color code.

$$
\begin{gathered}
H_{x}(z)=-A \frac{k}{\omega} \operatorname{sgn}(z) e^{i k|z|}, \\
H_{z}(z)=\frac{q}{\omega} A e^{i k|z|},
\end{gathered}
$$

and Eq. (C40) provides a secular equation for the SPP mode,

$$
2 i k=-\omega^{2} \chi
$$

which can be written more explicitly, using Eq. (C5), as

$$
k+\omega \sigma(\omega)=0
$$

identical to Eq. (6). To obtain an approximate analytic solution of Eq. (C44), let us use the fact that $|\sigma(\omega)| \ll 1$ if the frequency is not too small. This is due to the fact that $\sigma$ is proportional to the fine-structure constant $\alpha$ which is a small number. Combining Eqs. (C20) and (C44), we obtain

$$
\omega \approx q+\frac{q}{2} \sigma^{2}(q), \quad k \approx-q \sigma(q)
$$

Extracting the real and imaginary parts, we then find

$$
\begin{gathered}
\omega^{\prime} \approx q, \quad \omega^{\prime \prime} \approx q \sigma^{\prime}(q) \sigma^{\prime \prime}(q), \\
k^{\prime} \approx-q \sigma^{\prime}(q), \quad k^{\prime \prime} \approx-q \sigma^{\prime \prime}(q) .
\end{gathered}
$$

Interestingly, $k^{\prime}<0$, since $\sigma^{\prime}(\omega)>0$, at least for a real frequency - the same as in TM polarization. This implies that the light in the SPP mode propagates both along and toward the graphene sheet. At the same time, the amplitude of the wave exponentially decreases (increases) with distance from the sheet when $\sigma^{\prime \prime}(\omega)<0\left(\sigma^{\prime \prime}(\omega)>0\right)$. We also see that the sign of $k^{\prime \prime}$ and $\omega^{\prime \prime}$ changes simultaneously at the lower threshold frequency as we discuss in detail in Sec. III C. 

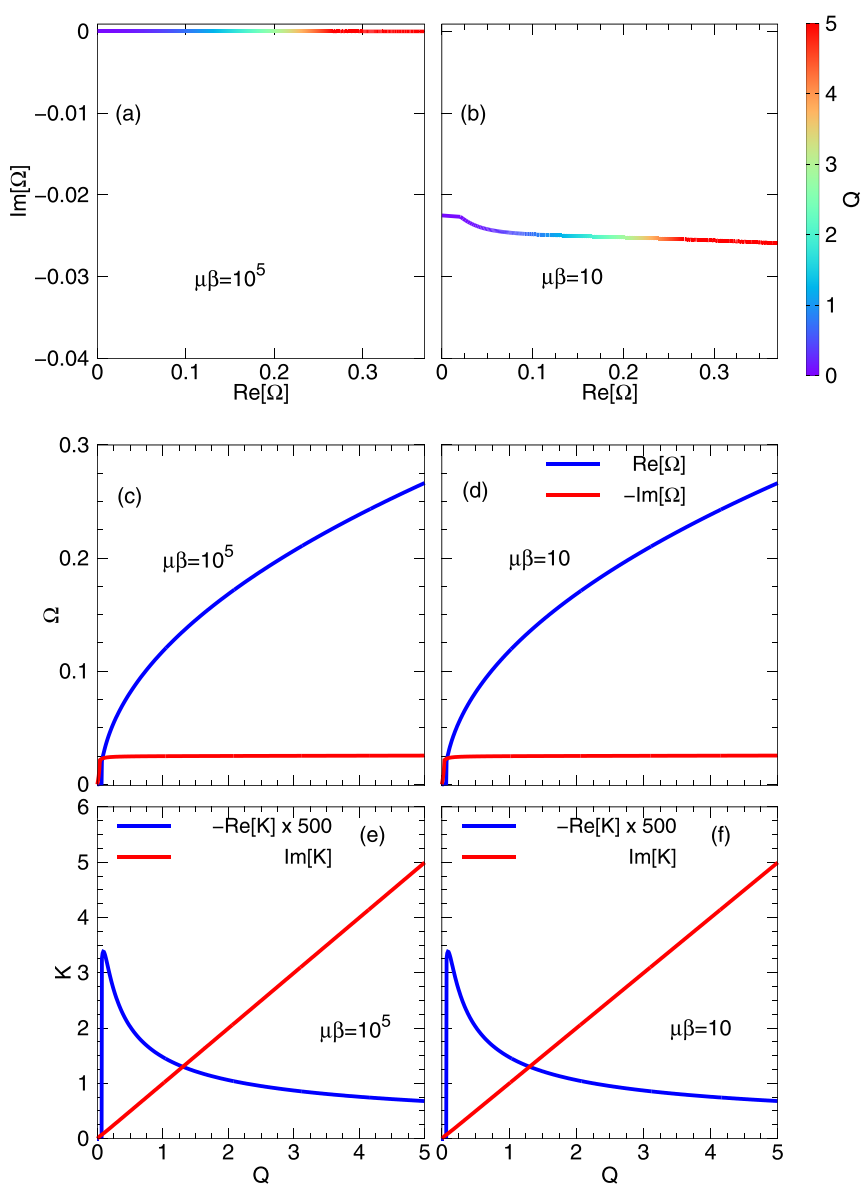

FIG. 6. As Fig. 2 but for $\Gamma=0.05$ and without using the analytic approximation.

\section{APPENDIX D: LOWER THRESHOLD FREQUENCY FOR TE POLARIZATION}

In this Appendix, we discuss in more detail the condition for the lower threshold frequency $\Omega_{\text {low }}^{\mathrm{TE}}$ of the TE mode and derive an equation determining the threshold value of $\Omega_{\text {low }}^{\mathrm{TE}} \approx$ 1.667 at zero temperature.

Let us first note that, in deriving Eq. (6), the electromagnetic field of the wave coupled to charge oscillations is proportional to

$$
e^{i k|z|}
$$

where $k=\sqrt{\omega^{2}-q^{2}}, q$ is the in-plane wave number in the direction of travel, and $z$ is distance to the graphene sheet. For a bounded solution, we therefore assert that

$$
\operatorname{Im} k>0 .
$$

In the opposite case, $\operatorname{Im} k<0$, the electromagnetic field would grow exponentially away from the graphene layer, and the threshold frequency is defined as a value at $\operatorname{Im} k=0$. As an example for finite temperature $\mu \beta=10$, we see in Fig. 5 that the signs of both $\operatorname{Im} k$ and $\operatorname{Im} \omega$ change simultaneously at the threshold frequency of $\Omega_{\text {low }}^{\mathrm{TE}} \approx 1.625$. This is in agreement with the analytic approximation given by Eqs. (9) and (10).

The value of the threshold frequency depends on the temperature (and the chemical potential) as demonstrated by
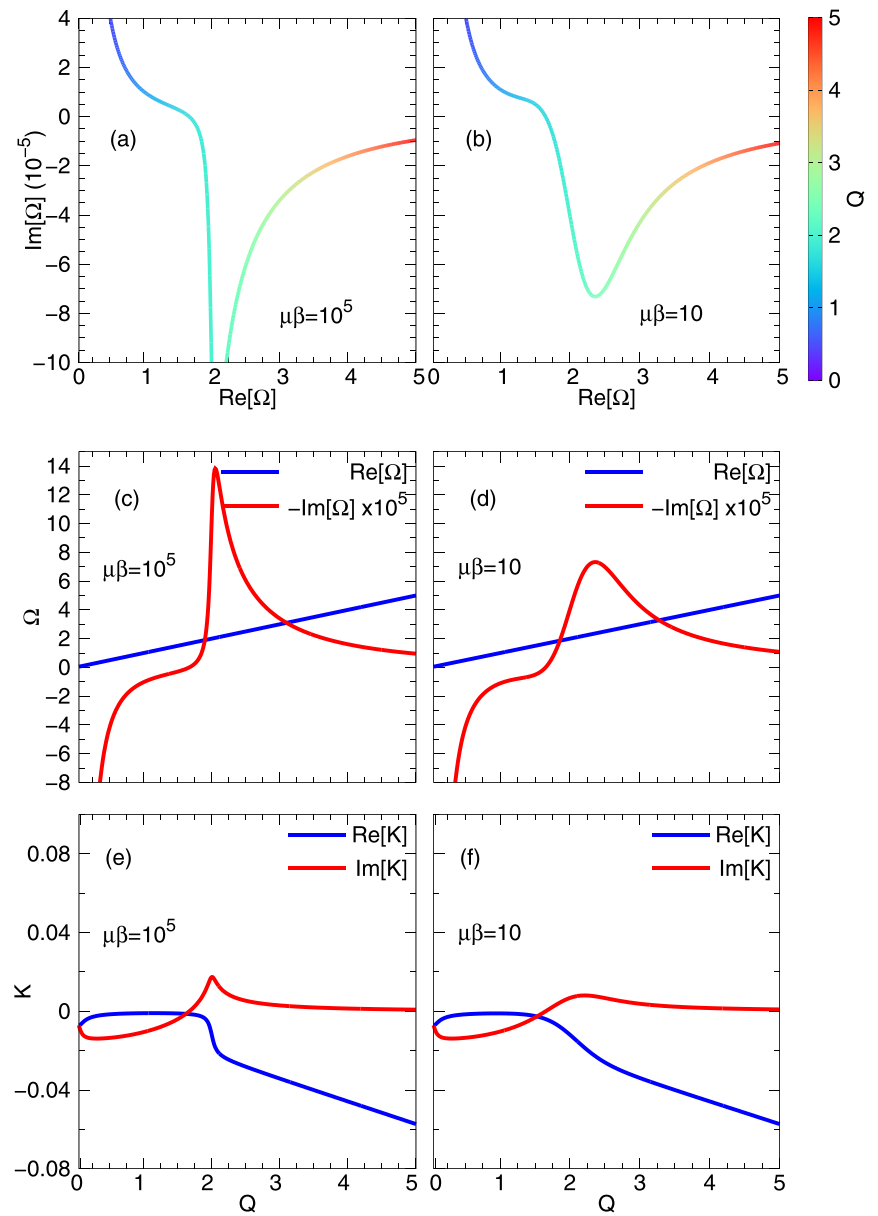

FIG. 7. As Fig. 3 but for $\Gamma=0.05$ and without using the analytic approximation.

Fig. 4. It satisfies a general equation

$$
\operatorname{Im} \sigma(\omega)=0,
$$

which can be easily obtained from Eq. (6) by using the fact that the mode frequency $\omega$ is real at the threshold, and hence $\operatorname{Im} k=0$. Then taking the imaginary part of Eq. (6) results in $k^{\prime \prime}+\omega \sigma^{\prime \prime}(\omega)=0$ which, in turn, gives Eq. (D3).

Finally, we consider the limit of zero temperature (and $\Gamma=$ 0 ), in which case the imaginary part of the conductivity takes the form

$$
\operatorname{Im} \sigma=\alpha\left(\frac{2}{\Omega}+\frac{1}{2} \ln \frac{2-\Omega}{2+\Omega}\right)
$$

following from Eq. (1), after performing the analytic integration in the interband part. Applying the threshold condition Eq. (D3) then leads to

$$
2+\Omega_{\mathrm{low}}^{\mathrm{TE}}=\left(2-\Omega_{\mathrm{low}}^{\mathrm{TE}}\right) \exp \left(\frac{4}{\Omega_{\mathrm{low}}^{\mathrm{TE}}}\right) .
$$

The numerical solution of this equation gives $\Omega_{\text {low }}^{\mathrm{TE}} \approx 1.667$. Figure 4 shows that $\Omega_{\text {low }}^{\mathrm{TE}}$ approaches this value in the limit $T \rightarrow 0$. 


\section{APPENDIX E: SPP MODES WITH NON-ZERO DAMPING}

In this Appendix, we show the SPP dispersion relation with nonzero phenomenological damping for both TM and TE polarizations. Figures 6(a)-6(d) show the dispersion of TM SPP mode for $\Gamma=0.05$. Here we observe that, compared to zero damping, Im $\omega$ is mainly lifted up by $\Gamma / 2$ throughout the range of $q$. Whereas $\operatorname{Im} k$, shown as red curves in Figs. 6(e) and 6(f), is unchanged compared to the case of $\Gamma=0$ (Fig. 2), $\operatorname{Re} k$, shown as blue curves in the same figures, changes its behavior and its values significantly, increasing fast around $q=0$ and then gradually decreasing with $q$.

In contrast, the TE SPP mode shown in Figs. 7(a)-7(d) demonstrates more changes in $\operatorname{Im} \omega$. In comparison with the dispersion for zero $\Gamma$ (Fig. 3), the TE SPP mode now has smoother dependencies of $\operatorname{Im} \omega$ and $k . \operatorname{Im} k$, shown in Figs. 7(e) and 7(f), starts from a negative value at $q=0$, and the TE SPP mode frequency still has a positive imaginary part in the range below the threshold frequency $\Omega_{\text {low }}^{\mathrm{TE}}$, similar to what we have seen for $\Gamma=0$.
[1] H. Raether, Surface Plasmons (Springer, Berlin, 1988).

[2] F. H. Koppens, D. E. Chang, and F. J. García De Abajo, Graphene plasmonics: A platform for strong light-matter interactions, Nano Lett. 11, 3370 (2011).

[3] K. W.-K. Shung, Dielectric function and plasmon structure of stage-1 intercalated graphite, Phys. Rev. B 34, 979 (1986).

[4] K. S. Novoselov, A. K. Geim, S. V. Morozov, D. Jiang, Y. Zhang, S. V. Dubonos, I. V. Grigorieva, and A. A. Firsov, Electric field in atomically thin carbon films, Science 306, 666 (2004).

[5] O. Vafek, Thermoplasma Polariton within Scaling Theory of Single-Layer Graphene, Phys. Rev. Lett. 97, 266406 (2006).

[6] G. W. Hanson, Dyadic Green's functions and guided surface waves for a surface conductivity model of graphene, J. Appl. Phys. 103, 064302 (2008).

[7] M. Jablan, H. Buljan, and M. Soljačić, Plasmonics in graphene at infrared frequencies, Phys. Rev. B 80, 245435 (2009).

[8] J. Chen, M. Badioli, P. Alonso-González, S. Thongrattanasiri, F. Huth, J. Osmond, M. Spasenović, A. Centeno, A. Pesquera, P. Godignon, A. Zurutuza Elorza, N. Camara, F. J. G. de Abajo, R. Hillenbrand, and F. H. L. Koppens, Optical nano-imaging of gate-tunable graphene plasmons, Nature (London) 487, 77 (2012).

[9] S. Kim, M. S. Jang, V. W. Brar, Y. Tolstova, K. W. Mauser, and H. A. Atwater, Electronically tunable extraordinary optical transmission in graphene plasmonic ribbons coupled to subwavelength metallic slit arrays, Nat. Commun. 7, 12323 (2016).

[10] Y. Francescato, V. Giannini, and S. A. Maier, Strongly confined gap plasmon modes in graphene sandwiches and graphene-onsilicon, New J. Phys. 15, 063020 (2013).

[11] A. Y. Nikitin, F. Guinea, and L. Martin-Moreno, Resonant plasmonic effects in periodic graphene antidot arrays, Appl. Phys. Lett. 101, 151119 (2012).

[12] S. A. Mikhailov and K. Ziegler, New Electromagnetic Mode in Graphene, Phys. Rev. Lett. 99, 016803 (2007).

[13] X. Y. He, J. Tao, and B. Meng, Analysis of graphene TE surface plasmons in the terahertz regime, Nanotechnology 24, 345203 (2013).

[14] O. Kotov, M. Kol'chenko, and Y. E. Lozovik, Ultrahigh refractive index sensitivity of TE-polarized electromagnetic waves in graphene at the interface between two dielectric media, Opt. Express 21, 13533 (2013).
[15] X. Zhang, H. Hu, X. Lin, L. Shen, B. Zhang, and H. Chen, Confined transverse-electric graphene plasmons in negative refractive-index systems, npj 2D Mater. Appl. 4, 25 (2020).

[16] Y. V. Bludov, D. A. Smirnova, Y. S. Kivshar, N. M. R. Peres, and M. I. Vasilevskiy, Nonlinear TE-polarized surface polaritons on graphene, Phys. Rev. B 89, 035406 (2014).

[17] L. A. Falkovsky and S. S. Pershoguba, Optical far-infrared properties of a graphene monolayer and multilayer, Phys. Rev. B 76, 153410 (2007).

[18] A. F. Page, F. Ballout, O. Hess, and J. M. Hamm, Nonequilibrium plasmons with gain in graphene, Phys. Rev. B 91, 075404 (2015).

[19] A. F. Page, J. M. Hamm, and O. Hess, Polarization and plasmons in hot photoexcited graphene, Phys. Rev. B 97, 045428 (2018).

[20] L. A. Weinstein, Open Resonators and Open Waveguides (Golem Press, Boulder, CO, 1969).

[21] E. A. Muljarov, W. Langbein, and R. Zimmermann, BrillouinWigner perturbation theory in open electromagnetic systems, Europhys. Lett. 92, 50010 (2010).

[22] P. Lalanne, W. Yan, K. Vynck, C. Sauvan, and J.-P. Hugonin, Light interaction with photonic and plasmonic resonances, Laser Photonics Rev. 12, 1700113 (2018).

[23] S. V. Lobanov, W. Langbein, and E. A. Muljarov, Resonantstate expansion of three-dimensional open optical systems: Light scattering, Phys. Rev. A 98, 033820 (2018).

[24] T. Weiss and E. A. Muljarov, How to calculate the pole expansion of the optical scattering matrix from the resonant states, Phys. Rev. B 98, 085433 (2018).

[25] M. B. Doost, W. Langbein, and E. A. Muljarov, Resonant-state expansion applied to three-dimensional open optical systems, Phys. Rev. A 90, 013834 (2014).

[26] M. V. Rybin, K. L. Koshelev, Z. F. Sadrieva, K. B. Samusev, A. A. Bogdanov, M. F. Limonov, and Y. S. Kivshar, High- $q$ Supercavity Modes in Subwavelength Dielectric Resonators, Phys. Rev. Lett. 119, 243901 (2017).

[27] S. V. Lobanov, W. Langbein, and E. A. Muljarov, Resonantstate expansion applied to three-dimensional open optical systems: Complete set of static modes, Phys. Rev. A 100, 063811 (2019).

[28] C. Sauvan, J. P. Hugonin, I. S. Maksymov, and P. Lalanne, Theory of the Spontaneous Optical Emission of Nanosize Photonic and Plasmon Resonators, Phys. Rev. Lett. 110, 237401 (2013). 
[29] E. A. Muljarov and W. Langbein, Resonant-state expansion of dispersive open optical systems: Creating gold from sand, Phys. Rev. B 93, 075417 (2016).

[30] H. S. Sehmi, W. Langbein, and E. A. Muljarov, Applying the resonant-state expansion to realistic materials with frequency dispersion, Phys. Rev. B 101, 045304 (2020).

[31] L. J. Armitage, M. B. Doost, W. Langbein, and E. A. Muljarov, Resonant-state expansion applied to planar waveguides, Phys. Rev. A 89, 053832 (2014).

[32] S. G. Tikhodeev, A. L. Yablonskii, E. A. Muljarov, N. A. Gippius, and T. Ishihara, Quasiguided modes and optical properties of photonic crystal slabs, Phys. Rev. B 66, 045102 (2002).

[33] T. Weiss, M. Mesch, M. Schäferling, H. Giessen, W. Langbein, and E. A. Muljarov, From Dark to Bright: First-Order Perturbation Theory with Analytical Mode Normalization for Plasmonic Nanoantenna Arrays Applied to Refractive Index Sensing, Phys. Rev. Lett. 116, 237401 (2016).

[34] S. Neale and E. A. Muljarov, Resonant-state expansion for planar photonic crystal structures, Phys. Rev. B 101, 155128 (2020).
[35] V. P. Gusynin, S. G. Sharapov, and J. P. Carbotte, Unusual Microwave Response of Dirac Quasiparticles in Graphene, Phys. Rev. Lett. 96, 256802 (2006).

[36] L. A. Falkovsky, Optical properties of graphene, J. Phys.: Conf. Ser. 129, 012004 (2008).

[37] L. A. Falkovsky, Optical properties of graphene and IV-VI semiconductors, Phys. Usp. 51, 887 (2008).

[38] V. I. Fal'ko and D. E. Khmel'nitskii, What if a film conductivity exceeds the speed of light? Zh. Eksp. Teor. Fiz. 95, 1988 (1989) [Sov. Phys. JETP 68, 1150 (1989)].

[39] H. Bruus and K. Flensberg, Many-body Quantum Theory in Condensed Matter Physics (Oxford University Press, Oxford, 2004)

[40] T. Stauber, N. M. R. Peres, and A. K. Geim, Optical conductivity of graphene in the visible region of the spectrum, Phys. Rev. B 78, 085432 (2008).

[41] G. D. Mahan, Many-Particle Physics, 3rd ed. (Böhlau Verlag, Wien, 2012), pp. 377-428. 